\title{
Paroxysmal Supraventricular Tachycardia: A Complex Dilemma during Pregnancy
}

\author{
${ }^{1}$ Bhavna Durgaprasad Kumare, ${ }^{2}$ Anjali Kawathalkar, ${ }^{3}$ Nikita Ritesh Vijay
}

\begin{abstract}
The acute and chronic management of paroxysmal supraventricular tachycardia (PSVT) during pregnancy presents a challenging clinical situation as there are no evidence-based guidelines despite being the commonest arrhythmia found in pregnancy. We report a case of paroxysmal supraventricular tachycardia in a 25 years old antenatal woman with no organic heart disease, where she received verapamil followed by diltiazem as antiarrhythmics instead of adenosine for conversion into sinus rhythm. Since she had recurrent episodes in third trimester she received verapamil and metoprolol as prophylaxis with good fetal and maternal outcome. This case highlights the need to understand the complexities in diagnosis and management of paroxysmal supraventricular tachycardia during pregnancy.
\end{abstract}

Keywords: Adenosine, Paroxysmal supraventricular, Pregnancy, Verapamil.

How to cite this article: Kumare BD, Kawathalkar A, Vijay NR. Paroxysmal Supraventricular Tachycardia: A Complex Dilemma during Pregnancy. J South Asian Feder Obst Gynae 2015;7(1):44-47.

Source of support: Nil

Conflict of interest: None

\section{INTRODUCTION}

Pregnancy is an arrhythmogenic state in which heart rhythm disorders may appear for first time or there can be exacerbation of pre-existing condition. ${ }^{1}$ The hemodynamic, hormonal, autonomic and emotional changes of pregnancy and presence of underlying heart disease are factors that can lead to arrhythmias. ${ }^{1,2}$ Ectopic beats and nonsustained arrhythmias are encountered in more than $50 \%$ of pregnant women investigated for palpitations which are generally benign and well

\footnotetext{
${ }^{1,3}$ Assistant Professor, ${ }^{2}$ Associate Professor

${ }^{1-3}$ Department of Obstetrics and Gynecology, NKP Salve Institute of Medical Sciences and Research Center, Nagpur Maharashtra, India

Corresponding Author: Bhavna Durgaprasad Kumare Assistant Professor, Department of Obstetrics and Gynecology NKP Salve Institute of Medical Sciences and Research Center Nagpur, Maharashtra, India, Phone: 07104236290, e-mail: bhavnakumare@gmail.com
}

tolerated requiring no treatment. ${ }^{3,4}$ Though sustained arrhythmias are relatively rare during pregnancy (2-3/1000), they do require treatment if associated with hemodynamic instability. ${ }^{3-5}$ Paroxysmal supraventricular tachycardia (PSVT) is the commonest tachyarrhythmia found in pregnancy presenting with palpitations, shortness of breath and presyncope. However, these symptoms are quite frequent in pregnancy leading to either over or delayed diagnosis of the condition with its consequences. This case report highlights about the complexities in the diagnosis and management of paroxysmal supraventricular tachycardia.

\section{CASE REPORT}

A 25 years old primigravida, averagely built and nourished was referred from a private hospital at 35 weeks with recurrent episodes of palpitations and shortness of breath for multidisciplinary management. On admission, she was in stable condition with pulse rate of 122 beats per minute, respiratory rate of $22 /$ minute and blood pressure of 100/70 mm Hg. There was no history of chest pain or syncope at the time of admission or in past. There was no history of such episodes in first trimester or before pregnancy. There was no history of sudden unexplained death in the family. She was admitted with similar episode at 28 weeks in private nursing home where she was evaluated for her symptoms. A complete blood count, sugars, serum electrolytes, thyroid profile, serum calcium and phosphorus was found to be normal. Her cardiac echocardiography did not show any structural and functional heart disease. An urgent electrocardiogram reported it as sinus tachycardia with rate of 145 to 150 beats/minute. Since no cause was found for her symptom and tachycardia, she was given carotid sinus massage for conversion to sinus rhythm. Unfortunately, it did not work, so she received two doses of $5 \mathrm{mg}$ of IV verapamil at interval with transient response. Hence, she received IV diltiazem $12.5 \mathrm{mg}$ which led to conversion to sinus rhythm. Since slight exertion led to increase in heart rate, she was started on oral sustained release verapamil $120 \mathrm{mg} 12$ hourly, metoprolol $12.5 \mathrm{mg}$ and digoxin $0.25 \mathrm{mg}$ once a day. She was discharged in sinus rhythm with these three drugs. After 2 weeks, digoxin and metoprolol was stopped as she remained asymptomatic. 
At 35 weeks, she was referred to our institute when she developed another episode of palpitations, shortness of breath and fatigue. An immediate 12-lead electrocardiogram (ECG) showed a heart rate of 150 beats/minute, narrow QRS complexes (0.10s), normal PR interval and normal axis on admission. Her repeat $C B C$, thyroid profile, serum electrolytes, renal function test, serum calcium, magnesium were found to be normal. Echocardiography was negative for structural or functional heart disease with LVEF of $65 \%$. Her upper and lower limb Doppler revealed normal flow studies with no focus of thromboembolism. Since she was hemodynamically stable and, already on verapamil, a beta blocker, metoprolol (12.5 mg twice a day) was restarted after consultation with cardiologist. Fortunately, she responded to metoprolol and developed sinus rhythm (84-96 beats/ minute) within few hours of admission. She continued to remain asymptomatic at rest but had palpitations on exertion. Fetal monitoring demonstrated a reactive non-stress test and a normal obstetric ultrasound. She remained hospitalized till 38 weeks of pregnancy as she and her family was anxious because of her cardiac condition. Because of concerns that labor pains may exacerbate her cardiac symptom, it was decided to deliver her by cesarean section at 38 weeks of gestation. She delivered a healthy female baby of $2.6 \mathrm{~kg}$ by an elective cesarean section which was done under combined spinal epidural anesthesia. Adenosine was kept ready during the surgery to terminate unexpected episode of SVT. Fortunately, her intraoperative and postoperative was uneventful with no recurrence of SVT. Verapamil was discontinued in postpartum period as it crosses the breast milk and she was discharged in sinus rhythm on oral metoprolol $12.5 \mathrm{mg}$ once a day 10 days later.

\section{DISCUSSION}

Sustained supraventricular tachycardia (SVT) is a cardiac emergency during pregnancy which has the potential to cause hemodynamic compromise with fetal consequence by decreasing uterine blood flow. Paroxysmal SVT is defined as any tachyarrhythmia with a heart rate greater than 120 beats per minute. ${ }^{2}$ The term 'paroxysmal' describes an arrhythmia that begins and ends abruptly. ${ }^{2}$ It excludes subtypes of atrial fibrillation and flutter, as well as multifocal atrial tachycardia. ${ }^{6}$ The main mechanism for the development of PSVT in women with structurally normal heart is via reentry, most commonly atrioventricular nodal reentrant tachycardia (AVNRT) in 60\% and atrioventricular reentrant tachycardia (AVRT) in 30\%. ${ }^{6}$

Correct interpretation of 12-lead ECG is extremely important during evaluation of any tachyarrhythmia during pregnancy as several ECG parameters are altered in the course of pregnancy. ${ }^{1}$ PSVT typically produces regular narrow QRS complexes $(<0.12 \mathrm{~s})^{1}$ whereas ventricular tachycardia (VT) produces wide QRS complexes (>0.12 s) on ECG. It is very important to differentiate SVT from VT as SVT responds well to AV nodal blocking antiarrhythmic drugs, whereas women with VT will suffer from hemodynamic deterioration if these drugs are given inadvertently. Hence, an early cardiology consultation is recommended for accurate diagnosis of arrhythmia. ${ }^{6}$ Thyroid dysfunction, electrolyte imbalance, anemia, fever, anxiety, toxic drug use and thromboembolism should be ruled out before the diagnosis of PSVT is made. Echocardiography helps to rule out structural and functional heart disease.

Acute treatment of PSVT in pregnancy is similar to that of nonpregnant women. ${ }^{4}$ Several reports have described successful nonpharmacological and pharmacological treatments of SVT in pregnant patients who are stable. ${ }^{6}$ It is recommended that non-pharmacologic maneuvers (carotid sinus massage, Valsalva maneuver) be tried first before embarking on pharmacological treatment. ${ }^{7}$ There are no class A antiarrhythmic drugs available to be used in pregnancy and all commonly used drugs are regarded as potentially toxic to fetus hence, they should be avoided if possible during the first trimester of pregnancy. ${ }^{7}$ The major concerns with various antiarrhythmic drugs, if taken in second and third trimester are fetal bradycardia and growth defects; hence, the smallest recommended dose should be used initially with regular monitoring of maternal and fetal condition. ${ }^{7}$ Secondly, absorption, bioavailability and elimination of these drugs are affected due to several physiological changes in pregnancy (increased blood volume, decreased protein concentration, alteration in gastric secretion and motility and hormonal stimulation of liver enzymes), hence dose adjustment and careful monitoring of both mother and fetus is essential as these changes can vary in magnitude during different stages of pregnancy. ${ }^{8}$ This could be the reason for recurrence of PSVT in our case at 35 weeks of pregnancy when metoprolol was resumed for rate control.

If vagal maneuvers are not effective, adenosine (class C), is the drug of choice to control SVT for its speed of action, efficacy and safety profile. ${ }^{1}$ Adenosine (6-12 mg slow IV) is rapidly metabolized and ideally suitable for use during pregnancy with $90 \%$ success. Verapamil (class C), a calcium channel blocker, is as effective as adenosine in converting an SVT to sinus rhythm. ${ }^{2}$ It is the alternative if the tachycardia is not reverted or in asthamatic women, ${ }^{1}$ however, fetal distress has been associated with drug induced maternal hypotension. ${ }^{1}$ Even beta blockers, metoprolol/propranolol (class C) 
Table 1: Antiarrhythmic drugs

\begin{tabular}{|c|c|c|c|}
\hline Drugs (FDA class) & Safety profile in pregnancy & Listed complications & Breast feeding \\
\hline Adenosine $(\mathrm{C})$ & Safe to use in pregnancy & $\begin{array}{l}\text { Pregnant women responds to lower } \\
\text { doses }\end{array}$ & Safe as short half life \\
\hline $\begin{array}{l}\text { Beta-blockers } \\
\text { Metoprolol (C) }\end{array}$ & Extensive experience and safe & - & Safe \\
\hline Propranolol (C) & Extensive experience and safe & $\begin{array}{l}\text { Low weight, fetal apnea bradycardia, } \\
\text { hypoglycemia }\end{array}$ & Safe \\
\hline Sotalol (B) & Limited experience but safe & Transient fetal bradycardia & Safe \\
\hline Atenolol (D) & $\begin{array}{l}\text { Avoid in first trimester-can lead } \\
\text { to IUGR }\end{array}$ & $\begin{array}{l}\text { IUGR, bradycardia, apnea, } \\
\text { hypoglycemia and hyperbilirubinemia }\end{array}$ & Safe \\
\hline $\begin{array}{l}\text { Calcium channel blockers } \\
\text { Verapamil }(C)\end{array}$ & Safe & $\begin{array}{l}\text { Rapid injection-maternal } \\
\text { hypotension and fetal distress }\end{array}$ & Safe \\
\hline Diltiazem (C) & Too little experience to comment & $\begin{array}{l}\text { Skeletal abnormality, IUGR, fetal } \\
\text { death }\end{array}$ & Safe \\
\hline Digoxin (C) & Good safety profile & Miscarriage and fetal death in toxicity & Safe \\
\hline Amiodarone (D) & $\begin{array}{l}\text { Only in resistant or life-threatening } \\
\text { cases for short-term use }\end{array}$ & $\begin{array}{l}\text { If prolonged use - fetal hypo/ } \\
\text { hyperthyroidism, goiter, IUGR, } \\
\text { prematurity }\end{array}$ & Avoid \\
\hline Flecainide (C) & Limited experience & $\begin{array}{l}\text { Insufficient data but no reported } \\
\text { complication }\end{array}$ & Unknown \\
\hline
\end{tabular}

can be used to control SVT. DC cardioversion is safe in all phases of pregnancy and should be considered in women who do not respond to pharmacotherapy and who are hemodynamically unstable. ${ }^{4}$ Fetal monitoring (by NST) and maternal monitoring (by ECG) during acute management of PSVT is paramount to diagnose fetal bradycardia and to see the response to maneuvers and pharmacotherapy respectively. ${ }^{1,2}$

Prophylactic treatment is restricted for women with poorly tolerated symptoms, recurrent SVT or if associated with hemodynamic compromise. ${ }^{1}$ Safety profile of antiarrhythmic drugs used in SVT during pregnancy and lactation are briefly summarized in Table $1 .^{3}$ Beta blockers, such as propranolol or metoprolol are the first choice and if they fail then, sotalol (class B), flecainide are recommended as monotherapy. ${ }^{1}$ If symptomatic episodes persist, combined treatment should be planned. ${ }^{1}$ Atenolol and amiodarone are FDA class D drugs and are avoided during pregnancy. Cathetor ablation should be recommended in symptomatic women before they contemplate pregnancy. ${ }^{8}$ During pregnancy, this procedure is restricted to drug refractory, poorly tolerated SVT and should be performed in second trimester if needed. ${ }^{1,8}$

Tocolytics (ritodrine, isoxsuprine) and oxytocin have been implicated to precipitate PSVT. ${ }^{2}$ Recommendations are that oxytocin should be given as bolus of 5 units maximum and administered slowly or as infusion especially in the presence of cardiovascular compromise. ${ }^{2}$ Vaginal delivery is not contraindicated in women with PSVT, however, emergency Cesarean section is often a consequence if the arrhythmia is intractable and resistant to medical management and DC cardioversion. ${ }^{2,9}$
In our case, either unavailability of adenosine or inexperience in using it might be the reasons for not using it as a first line drug in acute management of PSVT. However, the woman required two antiarrhythmic drugs, verapamil and diltiazem (both calcium channel blocker) for acute control of PSVT, suggesting partial success with verapamil.

\section{CONCLUSION}

Even though PSVT/SVT is the commonest arrhythmia in pregnancy, the degree of comfort and experience among obstetrician in treating it is minimal. So, when a pregnant woman with SVT presents to her obstetrician, assessment of vital signs, obtaining a 12-lead ECG, establishment of IV access, providing left lateral position, administration of $100 \%$ oxygen and frequent assessment of maternal symptoms and fetal stability are very important steps. Early cardiology consultation is recommended for correct diagnosis and management. Lastly, the knowledge about the various antiarrhythmic drugs that can be used during pregnancy and lactation is essential for favorable outcome. This case also highlights the need for keeping adenosine and verapamil in labor room as emergency drugs.

\section{REFERENCES}

1. Carlos DPV, Diego PD. Arrhythmias in pregnancy. Emergencias 2013;25(5):397-408.

2. Robins K, Lyons G. Supraventricular tachycardia in pregnancy. Br J Anaesth 2004;92(1):140-143.

3. Adamson DL, Nelson PC. Managing palpitations and arrhythmias during pregnancy. Heart 2007;93(12):1630-1636. 
4. Yilmaz F, Beydill I, Kavalci C, Yilmaz S. Successful electrical cardioversion of supraventricular tachycardia in a pregnant patient. Am J Case Rep 2012;13:33-35.

5. Tromp CH, Nanne ACM, Pernet PJM, Tukkie R, Bolte AC. Electrical cardioversion during pregnancy: safe or not? Neth Heart J 2011;19(3):134-136.

6. Ghosh N, Luk A, Derzko C, Dorain P, Chow CM. The acute treatment of maternal supraventricular tachycardias during pregnancy; a review of the literature. J Obstet Gynaecol Can 2011;33(1):17-23.
7. Tak T, Berkseth L, Maizer R. A case of supraventricular tachycardia associated with Wolff-Parkinson-White Syndrome and Pregnancy. WMJ 2012;111(5):228-232.

8. Blomström-LC, Scheinman MM, Aliot EM, Alpert JS, Calkins H, Camm J et al. ACC/AHA/ESC Guidelines for Management of SVA. J Am Coll Cardiol 2003;42(8):1493-1531.

9. Kyoung Ok Kim, Eun-Jung Chang, Jin Han, Hun Cho. Emergency cesarean delivery in a parturient who had an intractable paroxysmal supraventricular tachycardia: A case report. Korean J Anesthesiol 2012 Oct;63(4):360-362. 